\section{FedUni ResearchOnline \\ https://researchonline.federation.edu.au}

Copyright Notice

This is the peer-reviewed version of the following article:

Petty, Mark, Teng, Shyh, \& Murshed, Manzur. (2019). Improved image analysis methodology for detecting changes in evidence positioning at crime scenes.

Copyright @ 2020 IEEE. Personal use of this material is permitted. Permission from IEEE must be obtained for all other uses, in any current or future media, including reprinting/republishing this material for advertising or promotional purposes, creating new collective works, for resale or redistribution to servers or lists, or reuse of any copyrighted component of this work in other works. 


\title{
Improved Image Analysis Methodology for Detecting Changes in Evidence Positioning at Crime Scenes
}

\author{
Mark Petty, Shyh Wei Teng, and Manzur Murshed \\ School of Science, Engineering and Information Technology, Federation University Australia, Churchill, Victoria 3844, Australia \\ \{m.petty, shyh.wei.teng, manzur,murshed\}@federation.edu.au
}

\begin{abstract}
This paper proposed an improved methodology to assist forensic investigators in detecting positional change of objects due to crime scene contamination. Either intentionally or by accident, crime scene contamination can occur during the investigation and documentation process. This new proposed methodology utilises an ASIFT-based feature detection algorithm that compares pre- and post-contaminated images of the same scene, taken from different viewpoints. The contention is that the ASIFT registration technique is better suited to real world crime scene photography, being more robust to affine distortion that occurs when capturing images from different viewpoints. The proposed methodology was tested with both the SIFT and ASIFT registration techniques to show that (1) it could identify missing, planted and displaced objects using both SIFT and ASIFT and (2) ASIFT is superior to SIFT in terms of error in displacement estimation, especially for larger viewpoint discrepancies between the pre- and post-contamination images. This supports the contention that our proposed methodology in combination with ASIFT is better suited to handle real world crime scene photography.
\end{abstract}

Keywords - Image registration, change detection, crime scene image analysis

\section{INTRODUCTION}

Due to the rapid advancement of modern imaging and computer vision technologies, there has been an increase of the use of such technologies in crime scene investigation (CSI). A primary objective of using such technologies in CSI is to increase the accuracy and efficiency of investigating and documenting a crime scene, while protecting the integrity of the evidence.

The accurate preservation and representation of crime scenes ensures that evidences and their relative positions are available to investigators and the courts when upholding the rule of law. The immense pressure on investigators to process crime scenes in a timely and efficient manner could result in evidence contamination due to accidental human error or deliberate malicious interference. Such evidence contamination can harm the integrity of the crime scene and be detrimental to the investigation.

Defence lawyers are shifting their focus from the crime laboratory to the point of evidence collection. They suggest that where a piece of evidence has been improperly collected or tampered with, a petition to exclude the evidence from the trial proceedings might be successful. This may alter the outcome of the proceedings and lead to an unjust verdict.

If a contamination event is detected at an early stage, corrective measures can be taken before it is presented in court proceedings. However, like most processes, early fault detection can only occur if the overall process is being monitored.

To assist the CSI investigators to more effectively maintain the integrity of evidence collection on the crime scene, Abate et al. [1] recently proposed a change detection analysis methodology to detect crime scene contamination, through the use of photogrammetry techniques, such as feature based image registration. This methodology captures pre- and post-contaminated crime scene images and performs a comparison to detect mishandling of evidence during the investigation of the crime scene.

The primary limitation to Abate's methodology is their narrow definition of evidence contamination. Their methodology was restricted to the detection of missing objects. Whereas, a more realistic contamination scenario should also consider evidence that has been dislocated within the scene or added to the scene (planted).

Additionally, a more robust approach would also compare pre- and post-contaminated images from different viewpoints. Their methodology is limited to the comparison of images captured from viewpoints with only minor misalignment. This is due to the limitations of their SIFT-based corresponding feature keypoint matching technique [2]. SIFT, despite being invariant to changes in scaling, orientation and illumination, is only partly invariant to affine distortion which could occur when misaligned images are compared [3]. An instance of contamination which has been confirmed from numerous viewpoints, would dramatically increase the confidence in any practical methodology and reduce the instance of Types I and II errors.

Lastly, the evaluation of the effectiveness and robustness of Abate's methodology in [1] is limited to a qualitative analysis of images and a qualitative comparison with another 'developed' methodology. The effectiveness of any methodology could be better evaluated through a quantitative analysis.

Our proposed methodology aims to address the abovementioned limitations by detecting evidences, which are missing, dislocated and planted in pre- and post-contaminated images captured from different viewpoints. This will be achieved through image registration techniques, which are facilitated by substituting the feature-based registration technique from SIFT to ASIFT [4]. ASIFT is an extension of SIFT, that has been modified to be invariant to the affine distortion.

Additionally, our methodology will provide an estimation of the displacement of an object; a measurement of the distance an object has been moved within the scene. This will allow benchmarking against a measured distance and the 


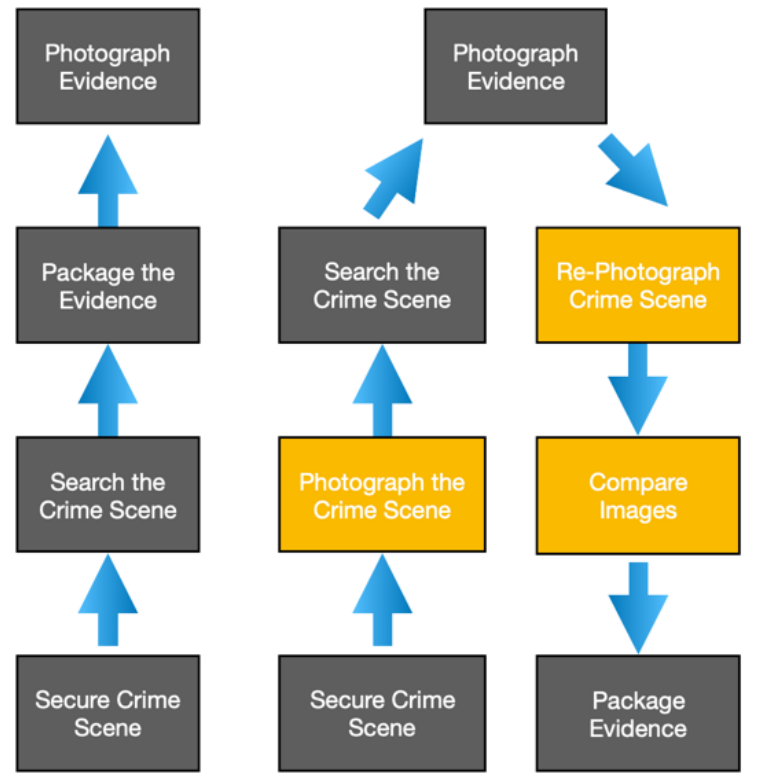

Fig. 1. (LHS) The traditional crime scene instigation process. (RHS) The modified crime scene investigation process proposed by Abate $e t$ al. [1].

calculation of the error rate of the methodology.

Our methodology, using ASIFT, was tested using both Abate's and our datasets to successful identify both missing and planted objects. Moreover, SIFT and ASIFT were incorporated in our proposed methodology during evaluation to identify and estimate displaced objects. This allowed a comparison of the two registration techniques that found that ASIFT is superior to SIFT in terms of error in displacement estimation, especially for larger viewpoint discrepancies between the pre- and post-contamination images.

The remainder of this paper is organised as follows: Section II describes related research works. Section III presents our proposed methodology. In Section IV, we will present experimental setup. Sections V and VI discuss our experimental results and an analysis of our proposed methodology's performance respectively. Finally, Section VII concludes the paper.

\section{RELATED WORKS}

\section{A. SIFT based contamination detection}

The methodology proposed by Abate et al. [1] is based on the approach that evidence contamination could be detected if the search stage of the traditional CSI process, see Fig. 1 (LHS), was monitored by recording the crime scene before and after the physical search, then comparing the pre- and post-search images for differences, see Fig. 1 (RHS).

Thus, Abate's proposed methodology can be summarised as follows:

- Feature points are extracted from both the pre- and post-contamination images using $\mathrm{SIFT}^{++}$[5], a variation of the conventional SIFT algorithm [3], and compared to find matching point correspondences. Using the $2 \mathrm{D}$ positions of each pair, a distance map is created to find the changed objects.

- Thresholding was then performed to compensate for image misalignment, due to the minor difference in pre and post image capture positioning.
- Areas where the change has been detected are masked and further RANSAC-verified feature matching [6] is completed against image templates of 'evidence images'.

- A geometric transform is estimated between the precontamination image and the matched 'evidence image' and used to register the two images.

Verification is performed by processing the images with another 'developed methodology', based on Multivariate Alteration Detection (MAD) and Maximum Autocorrelation Factor (MAF) techniques and comparing the results of the two methodologies. As the MAD-MAF methodology is not the primary technique, it is beyond the scope of this paper.

\section{B. Limitations of Abate Methodology}

Limitations in the experimental design of Abate, constrain how effective their methodology can be used in practice. These limitations are:

- Analysis-Only a limited evaluation of the effectiveness and robustness of their proposed approach is shown. The experimental process results in a qualitative analysis of images as opposed to a measurement-based approach, which would have allowed for a quantitative analysis, such as a statistical comparison of the results.

- Contamination type-The methodology only accounts for instances of missing objects. In each of the tests, a contamination event is simulated by the removal of one or more items represented in the precontamination image. Other equally likely contamination events are objects displaced from their original position or objects planted during the investigation stage. Both events could be equally damaging and should be taken into account. While it could be argued that Abate's methodology could also detect planted evidence by swapping the pre- and postcontamination images, an addition step would be required, as the detected 'planted' object would not match any of the 'evidence images'.

- Image misalignment-The pressure that crime scene investigators are under to record and collect evidence in a timely manner, makes it unlikely that they will have the time to photograph the scene using their proposed grid method. While photographers can attempt to capture pre- and post-contaminated images from the same location, due to human error, the possibility that the image viewpoint will differ must be taken into account. Their methodology compared preand post-contaminated images taken from approximately the same position. Any misalignment camera positioning was compensated for by using manual thresholding; 15-pixel segmentation. However, if their research was extended to consider increased misalignment between the images, an affine distortion would occur. This would challenge the SIFT registration technique that is being used for keypoint extraction and matching. SIFT, despite being invariant to changes in scaling, orientation and illumination, is only partly invariant to affine distortion [3]. Testing has shown that SIFT corresponding pairs decline after angles of only 30 degrees, completely failing after 50 degrees of affine angle [4, 7]. Any reduction in 


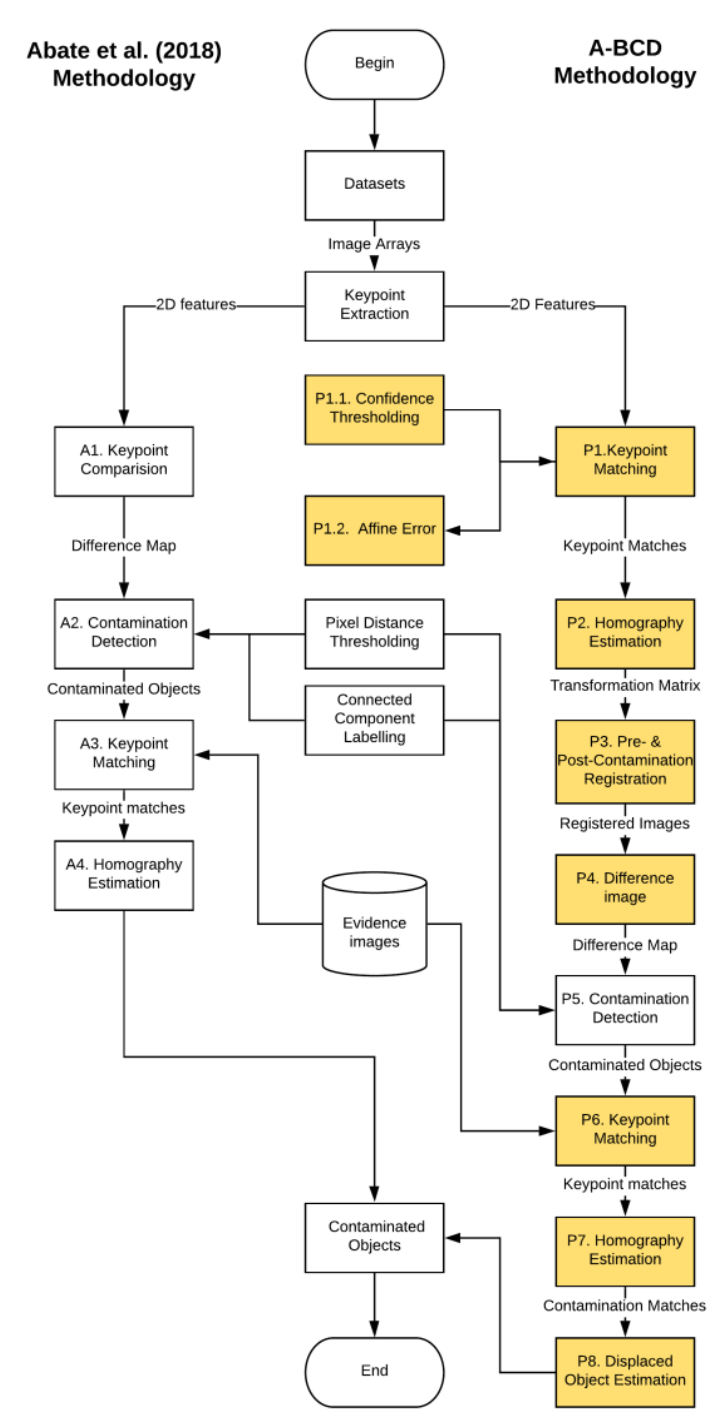

Fig. 2. (LHS) SIFT based methodology by Abate et al. [1]; (RHS) the proposed ASIFT based methodology.

corresponding feature pairs make image registration increasingly difficult [8].

\section{PROPOSED Methodology}

\section{A. ASIFT Feature Based Methodology}

Our proposed methodology improves the Abate's methodology by incorporating additional steps to detect both planted and missing objects, and to improve the robustness when processing misaligned images.

The modifications between the steps in our proposed methodology (P1...P8) and the steps in the Abate's methodology (A1...A4) are highlighted in Fig. 2 and are listed below:

- Whereas Abate only considers SIFT features (A1), we will consider ASIFT and SIFT Features (P1), in order to draw a comparison. Both feature sets will undergo confidence thresholding, set to $95 \%$, to control the possibility of a type I error; a false positive, see P1.1.

- The average Euclidean distance between the matching points are calculated (P1.2). This will be called the affine registration error and will be used to determine if the estimated object displacement (contamination) is due to errors in registration or to a genuine object displacement.

- Using the corresponding feature matches, a homographic estimation will be calculated, and a transformation matrix will be created (P2). The transformation matrix will be used to register the preand post-contamination images into a single fused image (P3).

- A difference image will be created (P4) from the fused image, which will undergo image segmentation, to isolate the areas of difference. These difference areas are then cropped to create cropped object images of the suspected contamination (P5).

- Feature matching (P6) between the cropped object images and the evidence images is performed, to determine if any of the known objects are present in both images. Matches are confirmed by estimating the homography (P7) and overlaying the images.

The type and extent of the contamination (P8) is then estimated by:

- If there is a match with an evidence image in either the pre- $\oplus$ post-contaminated image, then this can be identified as either a missing object or a planted object.

- If there is a match with an evidence image between the pre- and post-contaminated image, and the estimated distance is below the affine registration error, then this is identified as a non-contaminated object.

- If there is a match with an evidence image between the pre- and post-contaminated image, and the estimated distance is above the affine registration error, then this is identified as a contaminated object and the distance the object has moved is estimated.

\section{B. Estimation of Displaced Objects}

The proposed methodology estimates the displaced object distance by first determining the $2 \mathrm{D}$ coordinates of the upper RHS corner of the matched pairs of cropped object images bounding box. The coordinates are then used to calculate the Euclidean distance, in pixels between the two matching objects.

The measured distance is manually determined by measuring distance between the two instances of the object in the $0^{\circ}$ viewpoint registered image, using the MATLAB image measurement feature, imtool; part of the image processing toolbox.

\section{EXPERIMENTAL SETUP}

\section{A. Test Image Datasets}

Two image test datasets are used in this work for evaluating the performance of our proposed methodology. The first dataset comprises images from Groups A and C of Abate's test image dataset used in [1]. Group A comprises 42 images which represent a pre-contaminated crime scene with objects like flowers, gun, knife and can. Group C comprises 39 images of the same crime scene but it has been contaminated by removing the gun, knife and can (see images in Fig. 5 (LHS): top and second rows). We have also simulated a similar crime scene contamination scenario in which a book is removed from the simulated crime scene, as shown in Fig. 5 

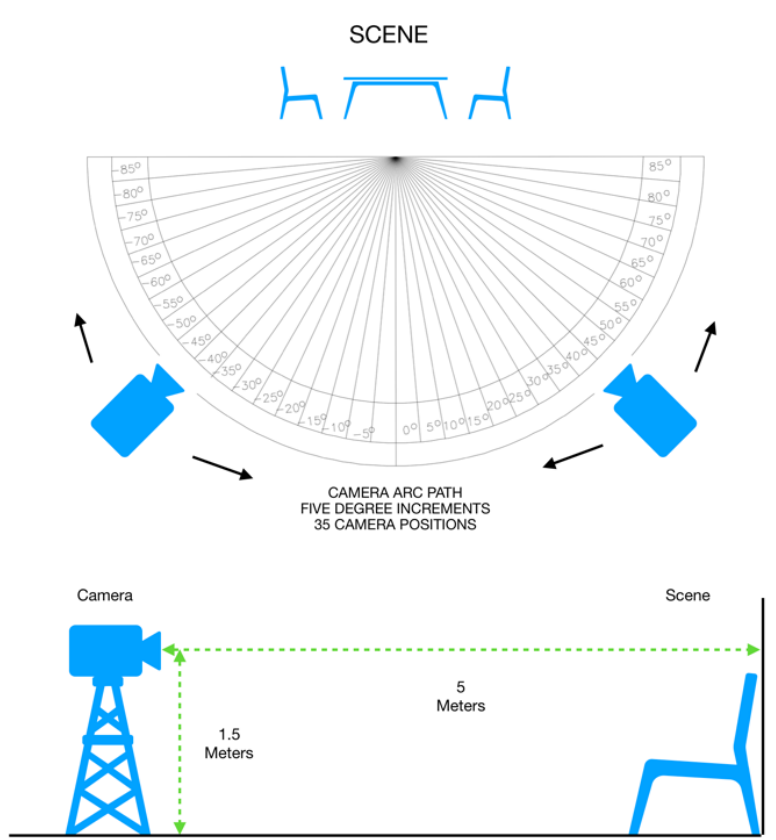

Fig. 3. Images groups captured at $5^{\circ}$ increments about the origin $\left(0^{\circ}\right)$, while maintaining focal length $(3.5 \mathrm{~m})$ and height $(1.8 \mathrm{~m})$.

(RHS): top and second rows. The images captured from this simulated crime scene are also included in this dataset.

The second test dataset comprises four groups of images captured from an indoor crime scene which we have simulated. The first group represents the pre-contaminated crime scene and it comprises 35 images captured at $5^{\circ}$ increments about the origin ( $0^{\circ}$ viewpoint ), while maintaining focal length $(3.5 \mathrm{~m})$ and height $(1.8 \mathrm{~m})$, as shown in Fig. 3. Fig. 4a shows the image of the pre-contaminated crime scene at $0^{\circ}$ viewpoint. The second group represents the crime scene with low level of contamination as the book moved slightly which is illustrated in Fig. 4b. This group also has 35 images as this contaminated crime scene is captured

(a) Pre-contamination (REFERENCE)

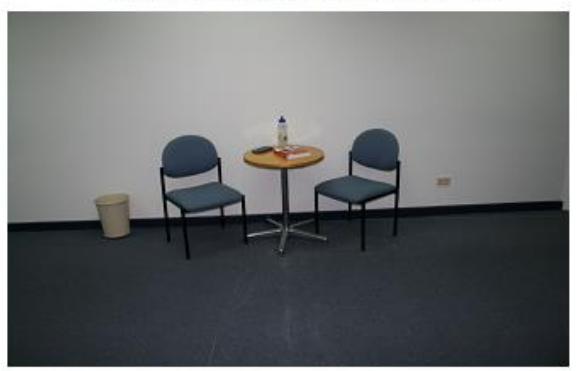

(c) MEDIUM contamination

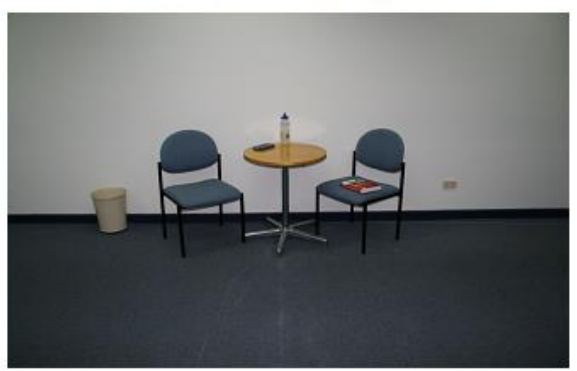

from all the 35 aforementioned viewpoints. The third and fourth groups are similar to the second group but these two groups represent the crime scene with medium and high levels of contamination, i.e. the book is moved at increasing distances respectively, as shown in Figs. 4c and $4 d$. An additional image was created of the $0^{\circ}$ viewpoint where the book was digitally cropped out of the image (Fig. 5 LHS middle) so that a comparison of missing or planted objects could be performed.

\section{B. Experiments}

The performance of our proposed methodology was evaluated using the two datasets described in Section IV.A. More specifically, we aim to evaluate the following:

- Equivalency of Abate's and our proposed methodologies: To test for missing or planted evidence, images that contained the simulated evidence objects were compared with images of the equivalent viewpoint, where the objects had been removed. This was performed with both Abates and our dataset.

- Displaced objects: To test for displaced objects and to estimate the degree of displacement, images from the reference group were compared to the LOW, MEDIUM and HIGH groups, using both SIFT and ASIFT.

\section{Camera Specifications}

Although the camera used in the experiment of Abate et al. [1] was a panoramic camera, our dataset was captured with an entry level digital camera, in order to represent a generic digital SLR camera [9] likely to be used by crime scene photographers, see Table I.

\section{Contaminated Object}

The contaminated object, shown in the fourth row of Fig. 6 , is the text book, 'Fast Track Visual Basic .Net' [10]. As our proposed methodology is based on feature keypoint detection,

(b) LOW contamination

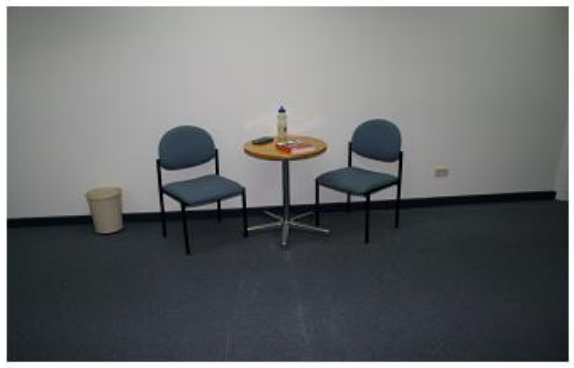

(d) HIGH contamination

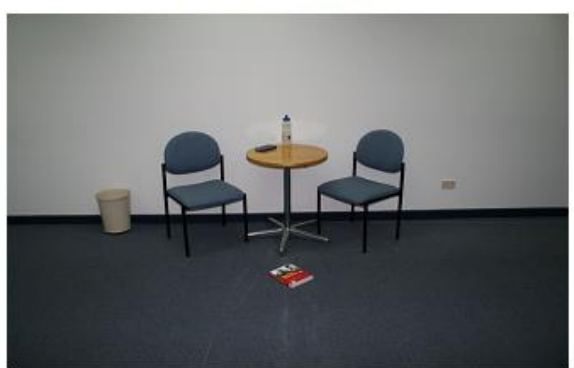

Fig. 4. Contaminated object position in image groups: (a) Reference image, (b) LOW contamination, (c) MEDIUM contamination and (d) HIGH contamination. 
TABLE I. TECHNICAL SPECIFICATION OF THE CANON EOS $400 D$

\begin{tabular}{lc}
\hline Property & Specifications \\
\hline Sensor Type & 10 MP APS-C CMOS \\
Sensor size (mm) & $22.2 \times 14.8$ \\
Image size (pixels) & $3888 \times 2592$ \\
Aperture & $\mathrm{f} / 4$ \\
Focal Length (mm) & 18 \\
Exposure & $1 / 60$ \\
ISO & $100-1600$ \\
\hline
\end{tabular}

an object with a high number of unique features was selected. This text book has a high number of robust feature keypoints on the cover and spine of the book, giving the methodology the best chance of success.
Objects with a low number of unique features would challenge both our proposed methodology and Abate's methodology. Thus, this challenge goes beyond the scope of this paper, however, could be addressed in future research.

\section{EXPERIMENTAL RESULTS}

\section{A. Performance of Proposed Methodology on Abate's}

Crime Scence Scenario

In the first set of experimental results, we aim to evaluate the performance of our proposed methodology on Abate's scenario whereby the crime scene is contaminated with objects are being removed. This experiment is conducted using the first dataset described in Section IV A. Fig. 5 shows an example of the detection outcome using the proposed methodology with ASIFT on both Abate's test images (RHS) and our test images (LHS). The top row shows the precontaminated images and the post-contaminated images are on the middle row. Displayed on the bottom row are the rectified images with the missing objects identified.
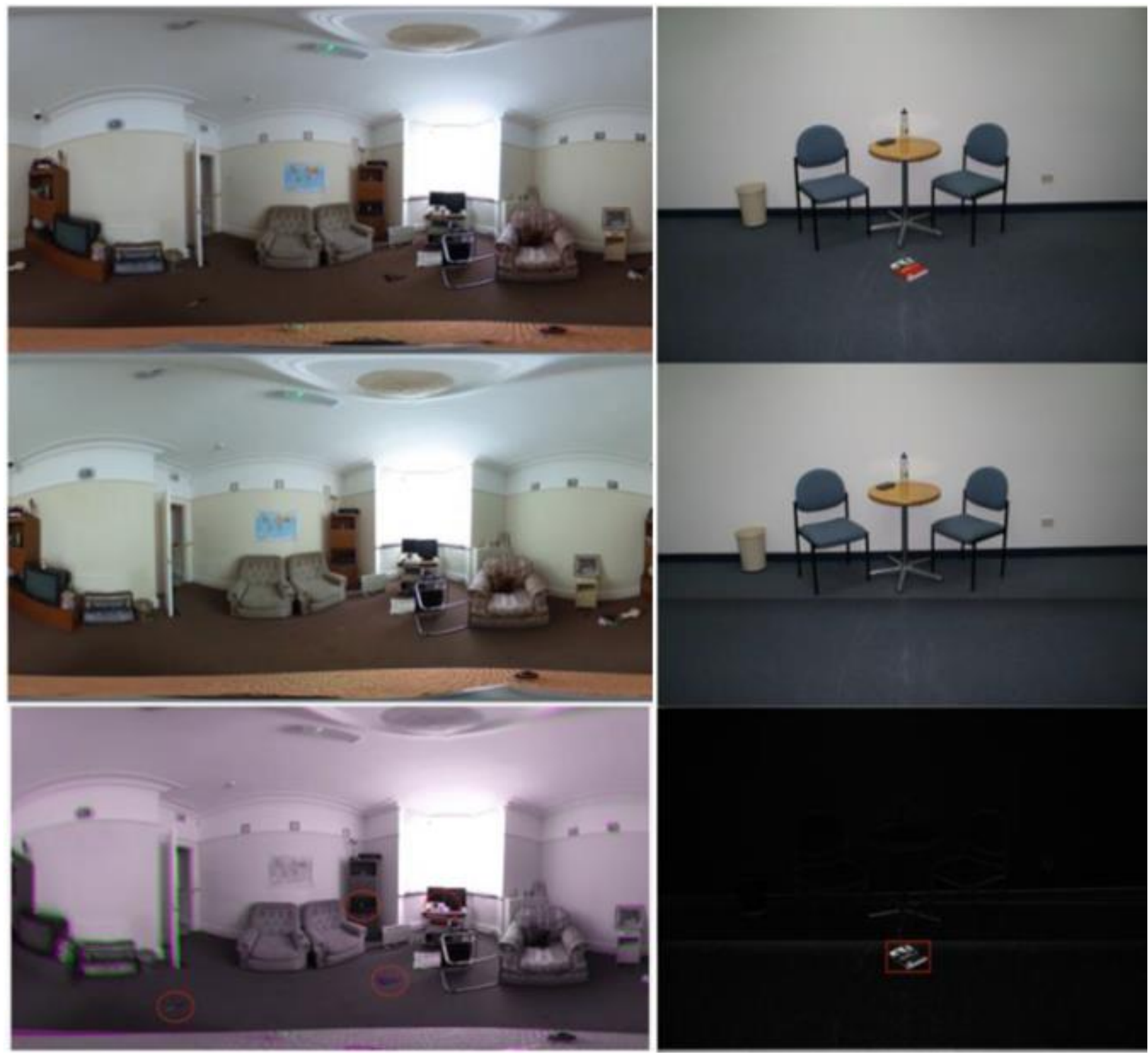

Fig. 5. (LHS) Abate's test images. (RHS) Our test images. First row pre-contamination image, second row post-contamination image. Third row missing items identified. 

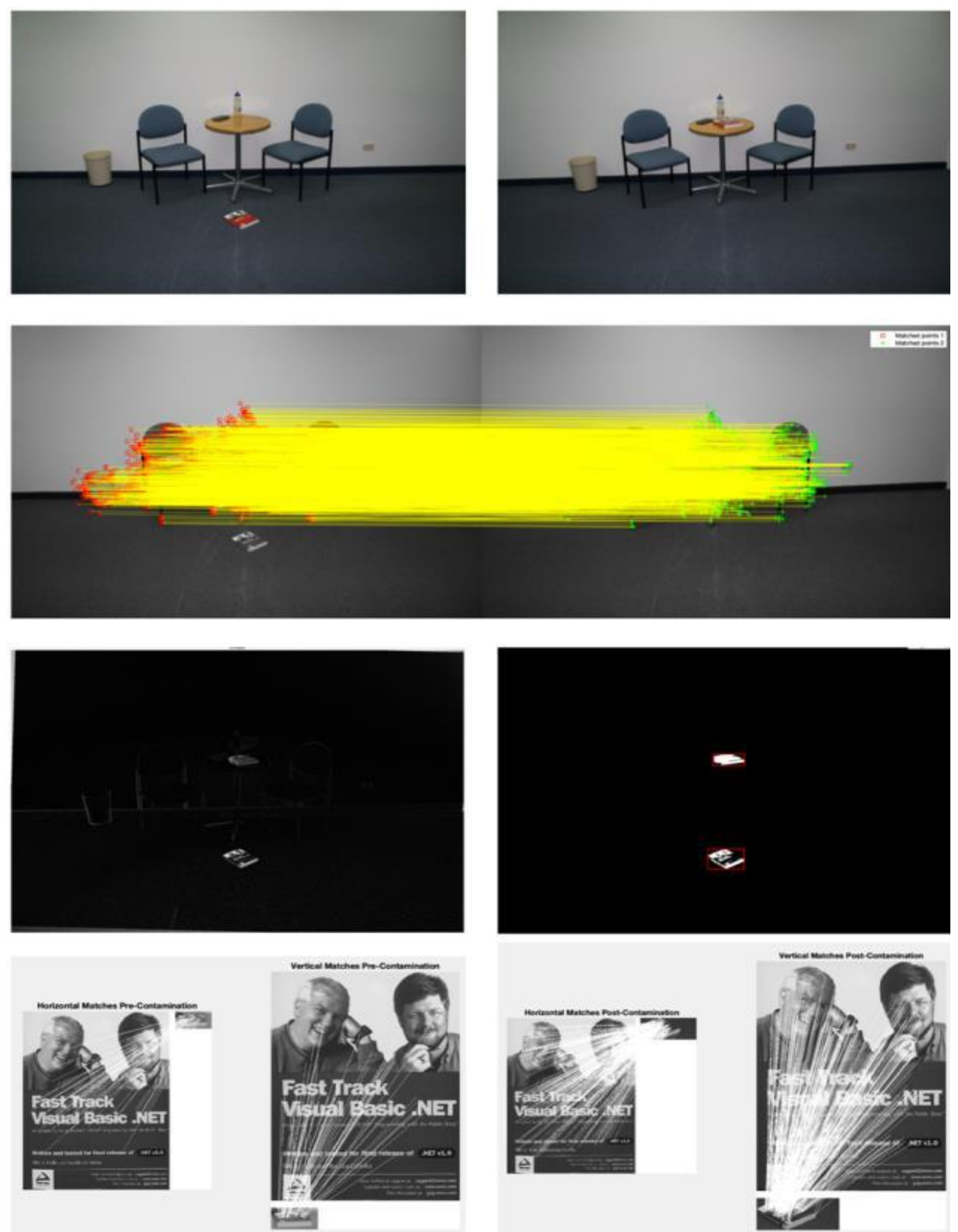

Fig. 6. Results of the proposed methodology. Top row - pre-(LHS), and post-contaminated (RHS) image. Second row - corresponding feature point matches. The third row - difference image (LHS), segmented image (RHS). The fourth row - cropped object and evidence image matches.

As expected, when the pre- and post-contaminated images were captured from the same viewpoint, with minor image misalignment, the proposed methodology with ASIFT has been able to successfully identify the missing and planted objects from both sets of images. Note that this shows that the performance of our proposed methodology is comparable to Abate's methodology for such a crime scene scenario.

\section{B. Identification Of Displaced Objects}

The identification of displaced objects using our proposed methodology, using both SIFT and ASIFT, was tested on the 
Number of matched keypoints between pre- and post-contamination images

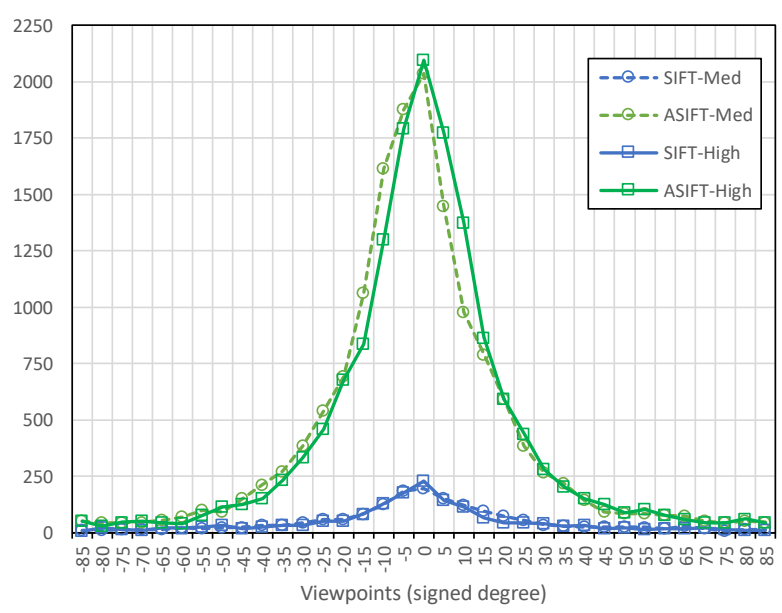

Fig. 7. SIFT and ASIFT matching pairs Vs image capture angle.

second dataset described in Section IV A. Fig. 6 shows an example of the proposed methodology successfully identifying the contaminated object on our dataset. Both the pre- and the post-contaminated images were captured from the same viewpoint. This result was also successfully replicated using the SIFT algorithm. The top row shows the pre- (LHS) and post-contaminated contaminated (RHS) images, whereas the second row shows the corresponding pairs between the two images. Note that there are no matches between the contaminated object on the floor and on the table, as the ASIFT algorithm filters the feature matches using RANSAC, which removes pairs that do not adhere to epipolar geometry.

The LHS of the third row shows the registered difference image, where the contaminated object has been highlighted in two locations (indicating a potential case for a displaced object.) The RHS of the same row shows the image segmentation result, identifying the areas to be cropped.

The bottom row shows the corresponding feature matches between the two cropped object images and the evidence image. This shows that there were two instances of the contaminated object in the registered image, proving that it has been displaced or contaminated between the pre-and postcontamination images.

\section{Displacment Estimation Of Objects}

Lastly, our proposed methodology was tested, using SIFT and ASIFT on estimating the degree of displacement of objects. Fig. 7 shows the number of corresponding matching pairs of keypoints in pre- and post-contaminated images in the datasets achieved by the proposed methodology using SIFT and ASIFT features at various viewpoints $\left(-85^{\circ}\right.$ and $85^{\circ}$ at $5^{\circ}$ intervals.) The results for images representing the MEDIUM and HIGH levels of contamination were comparable. Using ASIFT features, the number of matching pairs peaked with 2095 matches, and then declined exponentially with the viewpoint angle. Nevertheless, at least 50 matches were achieved in a wide range of viewpoints, $-55^{\circ}$ to $55^{\circ}$. Whereas using SIFT features, merely 167 matches were achieved at $0^{\circ}$ and even a short range of viewpoints, $-10^{\circ}$ to $10^{\circ}$, could not achieve more than 10 matches for all angles. Undoubtedly, ASIFT provides a much superior feature space to detect objects that are displaced far from the original position.
Average error in displacement estimation at the same absolute degree of viewpoints (-ve \& +ve)

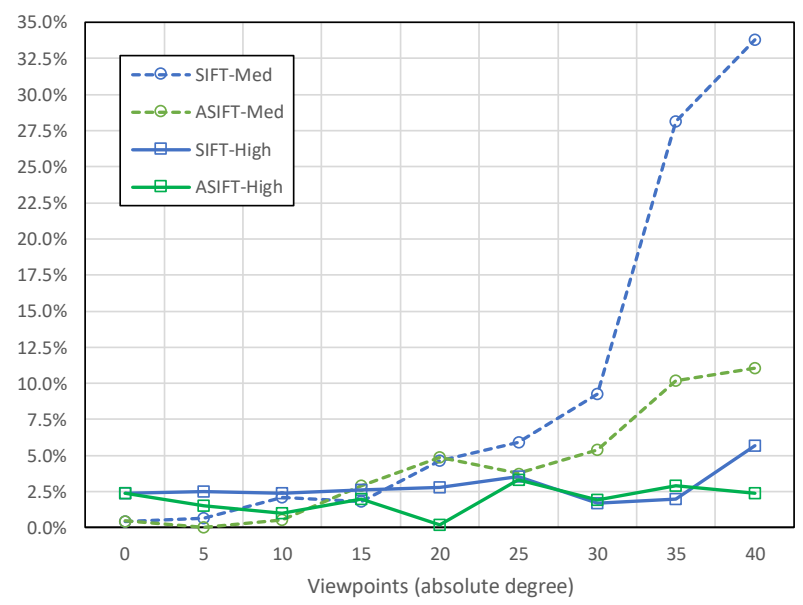

Fig 8. SIFT and ASIFT displacement estimation average error rates.

Fig. 8 outlines the average error in displacement estimation of the proposed methodology, using the SIFT and the ASIFT. The same absolute degree of viewpoints is considered, from $0^{\circ}$ to $40^{\circ}$ estimating the distance versus the measured distance, at $0^{\circ}$, of the MEDIUM and HIGH contaminated object change.

Clearly, ASIFT is superior to SIFT in terms of error in displacement estimation, especially for larger viewpoint discrepancies between the pre- and post-contamination images. As expected, the errors in both cases increase with the viewpoint discrepancies due to the diminishing number of matched keypoints.

The proposed methodology was not able to provide an estimation of the distance of the displacement of the contaminated object at LOW contaminated object change. Although the displacement was able to be measured using the MATLAB measurement tool, 20 pixels, the estimated degree of displacement was not greater than the affine registration error and was therefore rejected.

\section{Performance AnAlysis}

As illustrated Fig. 5, the proposed methodology was successfully able to reproduce the results of the Abate's methodology, using the ASIFT in place of the SIFT algorithm to identify missing contaminated objects. This was predicted, due to the similar performance of both algorithms in low affine distortion conditions [4,7]. Of additional interest, is the ability of the proposed methodology to operate on both panoramic and conventional images.

The successful identification of the displaced contaminated objects in Fig. 6, using both the SIFT and ASIFT algorithms, shows that it is possible to identify objects that have been displaced between the pre- and postcontamination images. This was predicted, as the proposed methodology relies on image registration to create a difference image between the two images, and successful image registration relies on a robust set of corresponding feature pairs, to perform homographic estimation and calculate a transformation matrix. Once again, this was predicted, due to the similar performance of both SIFT and ASIFT in low affine distortion conditions. 
Using the proposed methodology to estimate the degree of contamination of an object exposed the differences between SIFT and ASIFT. As shown in Fig. 8, as the viewpoint between the pre- and post-contamination images increases, so does the error rate between the measured and estimated object displacement. This is due to the diminishing number of matched keypoints that occur for both SIFT and ASIFT as the viewpoint increases, see Fig. 7.

Therefore, while it would appear that both SIFT and ASIFT can estimate the degree of displacement at viewpoints from $0^{\circ}$ to $40^{\circ}$, ASIFT is clearly superior to SIFT, due the lower error rate that it displays at higher viewpoints.

The inability to detect displacement of an object in the LOW group of images, highlighted a limitation in the proposed methodology. This was due to the relatively small object displacement of the LOW category, 20 pixels Vs MEDIUM 267 pixels and HIGH 476 pixels. Due to the errors that occur in image registration, the proposed methodology did not have the resolution to distinguish between small object displacements and image registration errors.

\section{CONCLUSIONS}

This paper proposes an improved image analysis methodology which will assist forensic investigators in crime scene preservation. The proposed methodology was tested by using SIFT, and then ASIFT to: (1) identify missing and planted objects, (2) identify displaced objects within a crime scene, and (3) to estimate the extent to which the contaminated objects have been moved.

From the experimental results presented, we have shown that our proposed methodology outperforms Abate's methodology significantly. This shows that compared to SIFT, ASIFT is able to reliably identify missing and displaced contaminated objects from pre- and post-contaminated images and was able to estimate the degree of displacement of the contaminated objects.

\section{ACKNOWLEDGMENT}

We would like to acknowledge the assistance of Dr. Dante Abate, at the Science and Technology in Archaeology and Culture Research Centre of the Cyprus Institute, for making available datasets utilized in Panoramic images, 2D featurebased and change detection methods for the documentation of contaminated crime scenes, [1].

This work is carried out as a part of the first author's honours research. He would like to thank his supervisors, second and third authors, for their guidance and continued support.

\section{REFERENCES}

[1] D. Abate, I. Toschi, C. Sturdy-Colls, and F. Remondino, "Panaramic images, 2D feature-based and change detection methods for the documentation of contaminated crime scenes," International Archives of the Photogrammetry, Remote Sensing \& Spatial Information Sciences, vol. 42, no. 2, 2018.

[2] G. Lv, S. W. Teng, and G. Lu, "A detector of structural similarity for multi-modal microscopic image registration," Multimedia Tools and Applications, vol. 77, no. 6, pp. 7675-7701, 2018.

[3] D. G. Lowe, "Distinctive image features from scale-invariant keypoints," International journal of computer vision, vol. 60, no. 2, pp. 91-110, 2004.

[4] J.-M. Morel and G. Yu, "ASIFT: A new framework for fully affine invariant image comparison," SIAM journal on imaging sciences, vol. 2, no. 2, pp. 438-469, 2009.

[5] Vedaldi. (2010, 2/6/2019). Available: http://www.vlfeat.org/

[6] M. A. Fischler and R. C. Bolles, "Random sample consensus: a paradigm for model fitting with applications to image analysis and automated cartography," Communications of the ACM, vol. 24, no. 6 , pp. 381-395, 1981.

[7] J. Wu, Z. Cui, V. S. Sheng, P. Zhao, D. Su, and S. Gong, "A Comparative Study of SIFT and its Variants," Measurement science review, vol. 13, no. 3, pp. 122-131, 2013.

[8] X. Wang, Y. Li, H. Wei, and F. Liu, "An ASIFT-based local registration method for satellite imagery," Remote Sensing, vol. 7, no. 6, pp. 7044$7061,2015$.

[9] CNET. (2006, 15/07/2019). Canon EOS 400D - digital camera Series Specs. Available: https://www.cnet.com/products/canon-eos-400ddigital-camera-ef-s-18-55mm-and-ef-55-200mm-lenses/

[10]FAST TRACK VB. Apress, 2002. 